\title{
Formal balls in fuzzy quasi-metric spaces
}

\author{
You $\mathrm{Gao}^{\mathrm{a}}$, Qingguo $\mathrm{Li}^{\mathrm{a}, *}$, Lankun Guo ${ }^{\mathrm{b}}$, Jialiang $\mathrm{Xie}^{\mathrm{c}}$ \\ ${ }^{a}$ College of Mathematics and Econometrics, Hunan University, Changsha, 410082, China. \\ ${ }^{b}$ College of Mathematics and Computer Science, Hunan Normal University, Changsha, 410012, China. \\ ${ }^{c}$ College of Science, Jimei University, Xiamen, 361021, China.
}

Communicated by S. Romaguera

\begin{abstract}
The notions of Yoneda completeness and Smyth completeness on fuzzy quasi-metric spaces are introduced and their relationship with other types of completeness including sequentially Yoneda completeness and bicompleteness are investigated. Then we use the standard Yoneda completeness to characterize the order-theoretical properties of the poset $\left(B X, \sqsubseteq_{M}\right)$ of formal balls in a fuzzy quasi-metric space $(X, M, \wedge)$. The results show that if $\left(B X, \sqsubseteq_{M}\right)$ is a dcpo, then $(X, M, \wedge)$ is standard complete and conversely, $\left(B X, \sqsubseteq_{M}\right)$ forms a dcpo provided that $(X, M, \wedge)$ is standard Yoneda complete. Particularly, in a fuzzy metric space, we clarify three types of completeness which can be characterized by the directed completeness of the related poset of formal balls. (C)2017 All rights reserved.
\end{abstract}

Keywords: Fuzzy quasi-metric space, Yoneda complete, standard Yoneda complete, Smyth complete, formal ball. 2010 MSC: 06A06, 54A40.

\section{Introduction}

The notion of fuzzy metric spaces based on continuous t-norms firstly appeared in [12] (briefly, KMfuzzy metric spaces) and later was modified in [3]. Afterwards, Gregori and Romaguera presented the concept of fuzzy quasi-metric spaces in [8] as a generalization of fuzzy metric spaces. In particular, it has been proved that the class of quasi-metrizable topological spaces and the class of fuzzy quasi-metrizable topological spaces agree with each other. These notions have been widely used in various papers devoted to fuzzy topology $[7,10,13,15,16,25,28]$. Meanwhile, much research has focused on investigating the interplay between fuzzy measures, domain theory and fuzzy metric spaces [14, 17, 18, 19, 20, 24, 26, 27].

The connection between fuzzy metric spaces and domain theory is an interesting topic for which the notion of completeness plays a crucial role. For instance, Ricarte [19] introduced the notion of standard completeness on KM-fuzzy metric spaces and proved that a KM-fuzzy metric spaces $(X, M, *)$ with the minimum t-norm $*=\wedge$ is standard complete, if and only if the associated poset (BX, $\left.{ }_{M}\right)$ of formal balls is a dcpo (even a continuous domain). Later on, Mardones-Pérez and de Prada Vicente [14] introduced

\footnotetext{
*Corresponding author

Email addresses: gaoyoumath@126.com (You Gao), liqingguoli@aliyun.com (Qingguo Li)
}

doi:10.22436/jnsa.010.02.30 
three approaches to deal with the interplay between completeness of KM-fuzzy metric spaces and directed completeness of the associated posets of formal balls. Recently, $\mathrm{Wu}$ and Yue [26] studied fuzzy partial metric spaces and showed that a fuzzy partial metric space is layered complete, if and only if the poset of formal balls is a dcpo.

Based on the results mentioned above, we focus this paper on studying the similar problem in fuzzy quasi-metric spaces. Actually, Ricarte and Romaguera [20] has considered this problem on KM-fuzzy quasi-metric spaces in the sense of Kramosil and Michálek [12] and gave a counterexample to show that the associated poset of formal balls is not a dcpo in the standard complete KM-fuzzy quasi-metric space. In the classical case of quasi-metric spaces, it has been proved that each Yoneda complete or Smyth complete quasi-metric space can be modelled by the poset of formal balls [1, 11, 23]. However, the role of these two types of completeness in the study of fuzzy quasi-metric spaces has not been made clear. Motivated by [6], in this paper we first adopt the left K-Cauchy net, which is "asymmetric Cauchy", to define the Yoneda completeness and Smyth completeness on fuzzy quasi-metric spaces. The relationship among these two types of completeness and other two types of completeness on fuzzy quasi-metric spaces appeared in $[5,6,9,22]$ are also discussed. Furthermore, we study the relationship between completeness of fuzzy (quasi-)metric spaces and directed completeness of the associated posets of formal balls. By means of standard Yoneda completeness, we give a sufficient condition under which the poset of formal balls is directed complete in a fuzzy quasi-metric space.

The content is arranged as follows: after some basic notions in Section 2, a new notion of Yoneda limits in a fuzzy quasi-metric space is introduced and the relationship among several types of completeness on fuzzy quasi-metric spaces are investigated in Section 3, and the connection between completeness of fuzzy quasi-metric spaces, particularly, completeness of fuzzy metric spaces and directed completeness of the associated posets of formal balls is studied in Section 4. Finally, in Section 5, some questions that we want to study in the future are stated.

\section{Preliminaries}

Let us recall that a continuous t-norm is a continuous function $*:[0,1] \times[0,1] \rightarrow[0,1]$ that satisfies the following conditions for every $a, b, c, d \in[0,1]$ :

(1) $a * b=b * a$;

(2) $\mathrm{a} *(\mathrm{~b} * \mathrm{c})=(\mathrm{a} * \mathrm{~b}) * \mathrm{c}$;

(3) $a * 1=a$;

(4) $a * b \leqslant c * d$ whenever $a \leqslant c$ and $b \leqslant d$.

Definition 2.1 ([8]). A fuzzy quasi-metric space is a triple $(X, M, *)$, where $X$ is a non-empty set, $*$ is a continuous t-norm and $M$ is a fuzzy set on $X \times X \times(0,+\infty)$ satisfying the following conditions for all $x, y, z \in X$ and $t, s>0:$

$($ Fqm-1): $M(x, y, t)>0$.

(Fqm-2): $M(x, y, t)=M(y, x, t)=1$, if and only if $x=y$.

(Fqm-3): $M(x, z, t+s) \geqslant M(x, y, t) * M(y, z, s)$.

$($ Fqm-4): $M(x, y, \bullet):(0,+\infty) \rightarrow(0,1]$ is continuous.

The concept of a fuzzy quasi-metric space considered in this paper is in the sense of George and Veeramani [3]. For a fuzzy quasi-metric space $(X, M, *)$, the fuzzy set $M$ is often called a fuzzy quasimetric on $X$. Each fuzzy quasi-metric $M$ on $X$ induces a $T_{0}$ topology $\tau_{M}$ on $X$, which has the family of all open balls $\left\{B_{M}(x, r, t): x \in X, r \in(0,1), t>0\right\}$ as a base, where $B_{M}(x, r, t)=\{y \in X: M(x, y, t)>1-r\}$ for all $x \in X, r \in(0,1)$ and $t>0$. 
A net $\left\{x_{i}\right\}_{i \in D}$ in $(X, M, *)$ converges to $x \in X$ with respect to the topology $\tau_{M}$, if and only if the net $\left\{M\left(x, x_{i}, t\right)\right\}_{i \in D}$ converges to 1 for all $t>0$ with respect to the usual topology on $(0,1]$, denoted by $\lim _{i \in D} M\left(x, x_{i}, t\right)=1$. In this case, we call $x$ a limit of $\left\{x_{i}\right\}_{i \in D}$ in the fuzzy quasi-metric space $(X, M, *)$.

Definition $2.2([3,8])$. A fuzzy metric space $(X, M, *)$ is a fuzzy quasi-metric space such that the fuzzy quasi-metric $M$ on $X$ satisfies the symmetry axiom: $M(x, y, t)=M(y, x, t)$ for all $x, y \in X$ and $t>0$. The fuzzy set $M$ of a fuzzy metric space $(X, M, *)$ is often called a fuzzy metric.

Remark 2.3.

(1) If $M$ is a fuzzy quasi-metric on $X$, then the fuzzy set $M^{-1}$ on $X \times X \times(0,+\infty)$ which is defined by $M^{-1}(x, y, t)=M(y, x, t)$ is also a fuzzy quasi-metric on $X$. Moreover, if we denote $M^{s}$ as the fuzzy set on $X \times X \times(0,+\infty)$ given by $M^{s}(x, y, t)=\min \left\{M(x, y, t), M^{-1}(x, y, t)\right\}$, then $M^{s}$ is a fuzzy metric on $X$.

(2) Let $(X, M, *)$ be a fuzzy quasi-metric space. There are other two natural topologies $\tau_{M^{-1}}$ and $\tau_{M^{s}}$ on $X$ induced by $M^{-1}$ and $M^{s}$, respectively. It is clear that $\tau_{M^{s}}=\tau_{M} \vee \tau_{M^{-1}}$. We say that a net $\left\{x_{i}\right\}_{i \in D}$ in $(X, M, *)$ converges strongly to $x \in X$, if it converges with respect to the topology $\tau_{M^{s}}$.

Example 2.4 $([2,8])$. Let $(X, d)$ be a quasi-metric space and $*$ be any continuous $t$-norm. If the fuzzy set defined on $X \times X \times(0,+\infty)$ is given by $M_{d}(x, y, t)=\frac{t}{t+d(x, y)}$, then the triple $\left(X, M_{d}, *\right)$ is a fuzzy quasimetric space. The fuzzy set $M_{d}$ on $X \times X \times(0,+\infty)$ is frequently called the standard fuzzy quasi-metric induced by $d$. Furthermore, the topology $\tau_{d}$ induced by $d$ coincides with the topology $\tau_{M_{d}}$ induced by the fuzzy quasi-metric $M_{d}$.

Next, we give some basic notions of generalized Cauchy nets and completeness for a fuzzy quasimetric space.

Definition $2.5([2,6])$. Let $(X, M, *)$ be a fuzzy quasi-metric space. A net $\left\{x_{i}\right\}_{i \in D}$ in $X$ is said to be:

(1) left (right) K-Cauchy, if for each $\varepsilon \in(0,1)$ and $t>0$, there exists $k \in D$ such that $M\left(x_{i}, x_{j}, t\right)>1-\varepsilon$ for all $k \leqslant i \leqslant j(k \leqslant j \leqslant i)$. The fuzzy quasi-metric space $(X, M, *)$ is left $K$-(sequentially) complete, if every left K-Cauchy (sequence) net has a limit $x \in X$ in $(X, M, *)$.

(2) bi-Cauchy, if for each $\varepsilon \in(0,1)$ and $t>0$, there exists $k \in D$ such that $M\left(x_{i}, x_{j}, t\right)>1-\varepsilon$ for all $i, j \geqslant k$. The fuzzy quasi-metric space $(X, M, *)$ is bicomplete, if every bi-Cauchy sequence in $(X, M, *)$ converges strongly.

The notion of left K-sequentially completeness was firstly introduced and investigated in [6]. The bicompleteness is one of the most important completeness theory for fuzzy quasi-metric spaces [2, 9]. Romaguera et al. solved the bicompletion problem of fuzzy quasi-metric spaces and convinced us in [22] that it is possible to construct satisfactory bicompleteness theory for fuzzy quasi-metric spaces.

The following facts will be frequently used later.

Proposition 2.6. Let $(X, M, *)$ be a fuzzy quasi-metric space. For a net $\left\{x_{i}\right\}_{i \in D}$ in $X$, we have that

(1) $\left\{x_{i}\right\}_{i \in D}$ is a bi-Cauchy net, if and only if it is a left K-Cauchy and right K-Cauchy net.

(2) If $\left\{x_{i}\right\}_{i \in D}$ converges strongly to some $x \in X$, then $\left\{x_{i}\right\}_{i \in D}$ is a bi-Cauchy net.

\section{Completeness on fuzzy quasi-metric spaces}

For a $T_{0}$ topological space $(X, \tau)$, the specialization order $\sqsubseteq_{\tau}$ on $X$ is defined by $x \sqsubseteq_{\tau} y$, if and only if $x \in \operatorname{cl}\{y\}$ for all $x, y \in X$, where $\operatorname{cl}\{y\}$ denotes the closure of $y$. In a fuzzy quasi-metric space $(X, M, *)$, the partial order $\varsigma_{\tau_{M}}$ can be characterized by the fuzzy quasi-metric $M$ itself. 
Proposition 3.1. Let $(X, M, *)$ be a fuzzy quasi-metric space. Then the following conditions are equivalent for any $x, y \in X:$

(1) $x \sqsubseteq \tau_{M} y$.

(2) $M(x, y, t)=1$, for all $t>0$.

(3) $M(x, z, t) \geqslant M(y, z, t)$, for all $z \in X$ and $t>0$.

(4) $M(z, y, t) \geqslant M(z, x, t)$, for all $z \in X$ and $t>0$.

The specialization order is an important notion which bridges $T_{0}$ topological spaces and ordered sets. It should be mentioned that the equivalence between (1) and (2) in Proposition 3.1 is well-known (for instance, see [21]) and the proof is omitted.

Proposition 3.2. Let $(X, M, *)$ be a fuzzy quasi-metric space and $\left\{x_{i}\right\}_{i \in D}$ be a net in $X$. If $\left\{x_{i}\right\}_{i \in D}$ converges strongly to $x \in X$, then $x$ is a largest limit of $\left\{x_{i}\right\}_{i \in D}$ in $(X, M, *)$ with respect to the specialization order $\Xi_{\mathrm{M}}$.

Proof. Since $\left\{x_{i}\right\}_{i \in D}$ converges strongly to $x \in X, x$ is clearly a limit of $\left\{x_{i}\right\}_{i \in D}$ in $(X, M, *)$. For each $\varepsilon \in(0,1)$, due to continuity of the t-norm $*$, there exists $\delta \in(0,1)$ such that $(1-\delta) *(1-\delta)>1-\varepsilon$. As $\left\{x_{i}\right\}_{i \in D}$ converges strongly to $x$ for each $t>0$, there exists $j_{1} \in D$ such that $M^{s}\left(x, x_{i}, t\right)>1-\delta$, in particular, $M\left(x_{i}, x, t\right)>1-\delta$ for all $i \geqslant j_{1}$.

Suppose that $y \in X$ is another limit of $\left\{x_{i}\right\}_{i \in D}$. Then there exists $j_{2} \in D$ such that $M\left(y, x_{i}, \varepsilon\right)>1-\delta$ for all $i \geqslant j_{2}$. Thus, there exists $j \geqslant j_{1}, j_{2} \in D$ such that

$$
M(y, x, t+\varepsilon) \geqslant M\left(y, x_{i}, \varepsilon\right) * M\left(x_{i}, x, t\right) \geqslant(1-\delta) *(1-\delta)>1-\varepsilon,
$$

for all $i \geqslant j$.

Let $\varepsilon \rightarrow 0$, we have $M(y, x, t)=1$ for all $t>0$. Therefore, $y \sqsubseteq \tau_{M} x$.

For a fuzzy quasi-metric space, the limit of a net is not always unique if it exists. In the sequel, we will introduce the notion of Yoneda limits of a net in a fuzzy quasi-metric space. Some related results in [4] can be properly extended to the fuzzy quasi-metric situation.

Definition 3.3. Let $\left\{x_{i}\right\}_{i \in D}$ be a net in a fuzzy quasi-metric space $(X, M, *)$. An element $x \in X$ is said to be a Yoneda limit of $\left\{x_{i}\right\}_{i \in D}$, if we have

$$
M(x, y, t)=\sup _{j \in D} \inf _{i \geqslant j} M\left(x_{i}, y, t\right), \quad \forall y \in X, \quad t>0 .
$$

Proposition 3.4. For a fuzzy quasi-metric space $(X, M, *)$, the Yoneda limit of a net $\left\{x_{i}\right\}_{i \in D}$ in $X$ is unique if it exists.

Proof. Assume that $z_{1}, z_{2} \in X$ are Yoneda limits of $\left\{x_{i}\right\}_{i \in D}$. Then for any $y \in X$ and $t>0$, we have

$$
M\left(z_{1}, y, t\right)=\sup _{j \in D} \inf _{i \geqslant j} M\left(x_{i}, y, t\right)=M\left(z_{2}, y, t\right) .
$$

Let $y=z_{1}$, then $1=M\left(z_{1}, z_{1}, t\right)=M\left(z_{2}, z_{1}, t\right)$. Similarly, let $y=z_{2}$, then $M\left(z_{1}, z_{2}, t\right)=M\left(z_{2}, z_{2}, t\right)=1$. Thus, $M\left(z_{2}, z_{1}, t\right)=M\left(z_{1}, z_{2}, t\right)=1$ for all $t>0$, which follows that $z_{1}=z_{2}$.

For a fuzzy quasi-metric space $(X, M, *)$, in order to avoid confusion, we call $x$ a $M^{-1}$-Yoneda limit and a $M^{s}$-Yoneda limit of a net $\left\{x_{i}\right\}_{i \in D}$, if $x$ is a Yoneda limit of $\left\{x_{i}\right\}_{i \in D}$ in $\left(X, M^{-1}, *\right)$ and $\left(X, M^{s}, *\right)$, respectively.

Lemma 3.5. Let $(X, M, *)$ be a fuzzy quasi-metric space and $\left\{x_{i}\right\}_{i \in D}$ be a net in $X$ satisfying that it converges strongly to $\mathrm{x} \in \mathrm{X}$. Then $\mathrm{x}$ is a Yoneda limit, a $\mathrm{M}^{-1}$-Yoneda limit and a $\mathrm{M}^{\mathrm{s}}$-Yoneda limit of $\left\{\mathrm{x}_{\mathrm{i}}\right\}_{\mathrm{i} \in \mathrm{D}}$. 
Proof. Since $\left\{x_{i}\right\}_{i \in D}$ converges strongly to $x \in X$, for each $\varepsilon \in(0,1)$ and $t>0$, there exists $j_{0} \in D$ such that

$$
M\left(x_{i}, x, \varepsilon t\right)>1-\varepsilon, \text { and } M\left(x, x_{i}, \varepsilon t\right)>1-\varepsilon,
$$

for all $i \geqslant j_{0}$.

For any $y \in X$, by the condition (Fqm-3),

$$
\begin{aligned}
M\left(x_{i}, y, t\right) & \geqslant M\left(x_{i}, x, \varepsilon t\right) * M(x, y,(1-\varepsilon) t) \\
& \geqslant(1-\varepsilon) * M(x, y,(1-\varepsilon) t),
\end{aligned}
$$

for all $i \geqslant j_{0}$. In particular, we get

$$
\inf _{i \geqslant j_{0}} M\left(x_{i}, y, t\right) \geqslant(1-\varepsilon) * M(x, y,(1-\varepsilon) t),
$$

which follows that

$$
\sup _{j \in D} \inf _{i \geqslant j} M\left(x_{i}, y, t\right) \geqslant \inf _{i \geqslant j_{0}} M\left(x_{i}, y, t\right) \geqslant(1-\varepsilon) * M(x, y,(1-\varepsilon) t) .
$$

Let $\varepsilon \rightarrow 0$, we have $\sup _{j \in D} \inf _{i \geqslant j} M\left(x_{i}, y, t\right) \geqslant 1 * M(x, y, t)=M(x, y, t)$.

For each $j \in D$, we can choose an $i_{0} \geqslant j_{0}, j$ by $D$ being directed, thus

$$
\begin{aligned}
M(x, y,(1+\varepsilon) t) & \geqslant M\left(x, x_{i_{0}}, \varepsilon t\right) * M\left(x_{i_{0}}, y, t\right) \geqslant(1-\varepsilon) * M\left(x_{i_{0}}, y, t\right) \\
& \geqslant \inf _{i \geqslant j}(1-\varepsilon) * M\left(x_{i}, y, t\right) \\
& \geqslant(1-\varepsilon) * \inf _{i \geqslant j} M\left(x_{i}, y, t\right) .
\end{aligned}
$$

Since $j \in D$ is arbitrarily chosen, we have

$$
M(x, y,(1+\varepsilon) t) \geqslant \sup _{j \in D}(1-\varepsilon) * \inf _{i \geqslant j} M\left(x_{i}, y, t\right)=(1-\varepsilon) * \sup _{j \in D} \inf _{i \geqslant j} M\left(x_{i}, y, t\right) .
$$

Let $\varepsilon \rightarrow 0$, then

$$
M(x, y, t) \geqslant 1 * \sup _{j \in D} \inf _{i \geqslant j} M\left(x_{i}, y, t\right)=\sup _{j \in D} \inf _{i \geqslant j} M\left(x_{i}, y, t\right) .
$$

Therefore, we obtain that $M(x, y, t)=\sup _{j \in D} \inf _{i \geqslant j} M\left(x_{i}, y, t\right)$ for all $y \in X$ and $t>0$, i.e., $x$ is a Yoneda limit of $\left\{x_{i}\right\}_{i \in D}$. It can be similarly proved that $x$ is also a $M^{-1}$-Yoneda limit and a $M^{s}$-Yoneda limit.

Lemma 3.6. If $x \in X$ is a Yoneda limit of a net $\left\{x_{i}\right\}_{i \in D}$ in a fuzzy quasi-metric space $(X, M, *)$, then $\left\{x_{i}\right\}_{i \in D}$ converges to $x$ with respect to the topology $\tau_{M^{-1}}$.

Proof. Fix $t>0$. Since $x$ is a Yoneda limit of $\left\{x_{i}\right\}_{i \in D}$, we know that

$$
M(x, y, t)=\sup _{j \in D} \inf _{i \geqslant j} M\left(x_{i}, y, t\right) \text { for all } y \in X
$$

Let $y=x$, then $1=M(x, x, t)=\sup _{j \in D} \inf _{i \geqslant j} M\left(x_{i}, x, t\right)$. For each $\varepsilon \in(0,1)$, there exists $j_{0}$ such that $\inf _{i \geqslant j_{0}} M\left(x_{i}, x, t\right)>1-\varepsilon$. Thus we have that $M\left(x_{i}, x, t\right)>1-\varepsilon$, for all $i \geqslant j_{0}$. Therefore, $\left\{x_{i}\right\}_{i \in D}$ converges to $x$ with respect to the topology $\tau_{M^{-1}}$.

Proposition 3.7. Let $\left\{x_{i}\right\}_{i \in D}$ be a net in a fuzzy quasi-metric space $(X, M, *)$. If $x$ is a $M^{-1}-$ Yoneda limit of $\left\{x_{i}\right\}_{i \in D}$, then $x$ is a largest limit of $\left\{x_{i}\right\}_{i \in D}$ in $(X, M, *)$ with respect to the specialization order $\sqsubseteq \tau_{M}$. 
Proof. By Lemma 3.6, $x$ is a limit of $\left\{x_{i}\right\}_{i \in D}$ in $(X, M, *)$. Suppose that $y$ is another limit of $\left\{x_{i}\right\}_{i \in D}$ in $(X, M, *)$. Then we have $\lim _{i \in D} M\left(y, x_{i}, t\right)=1$, in particular, $\sup _{j \in D} \inf _{i \geqslant j} M\left(y, x_{i}, t\right)=1$, for all $t>0$. Therefore,

$$
M(y, x, t)=M^{-1}(x, y, t)=\sup _{j \in D} \inf _{i \geqslant j} M^{-1}\left(x_{i}, y, t\right)=\sup _{j \in D} \inf _{i \geqslant j} M\left(y, x_{i}, t\right)=1 .
$$

Hence $y \sqsubseteq \tau_{M} x$.

Lemma 3.8. Let $\left\{x_{i}\right\}_{i \in D}$ be a left $K-C a u c h y$ net in a fuzzy quasi-metric space $(X, M, *)$. If $x \in X$ is a $M^{-1}-Y o n e d a$ limit of $\left\{x_{i}\right\}_{i \in D}$, then $\left\{x_{i}\right\}_{i \in D}$ converges strongly to $x \in X$.

Proof. Let $x$ be a $M^{-1}$-Yoneda limit of $\left\{x_{i}\right\}_{i \in D}$. Then $\left\{x_{i}\right\}_{i \in D}$ converges to $x$ with respect to the topology $\tau_{M}$ by Lemma 3.6. Now fix $t>0$. For each $\varepsilon \in(0,1)$, there exists $k_{1} \in D$ such that $M\left(x, x_{i}, t\right)>1-\varepsilon$, for all $i \geqslant k_{1}$.

Since $\left\{x_{i}\right\}_{i \in D}$ is left K-Cauchy, there exists $k_{2} \in D$ such that $M\left(x_{i}, x_{j}, t\right)>1-\varepsilon$, for all $j \geqslant i \geqslant k_{2}$. For each fixed $i \geqslant k_{2}$, it follows that $\inf _{j \geqslant i} M\left(x_{i}, x_{j}, t\right) \geqslant 1-\varepsilon$.

Then we conclude that

$$
\begin{aligned}
M^{-1}\left(x, x_{i}, t\right) & =\sup _{l \in D} \inf _{j} M^{-1}\left(x_{j}, x_{i}, t\right)=\sup _{l \in D} \inf _{j} M\left(x_{i}, x_{j}, t\right) \\
& \geqslant \inf _{j \geqslant i} M\left(x_{i}, x_{j}, t\right) \geqslant 1-\varepsilon,
\end{aligned}
$$

for each $i \geqslant k_{2}$.

Therefore, there exists $k \geqslant k_{1}, k_{2}$ such that

$$
M^{s}\left(x, x_{i}, t\right)=\min \left\{M\left(x, x_{i}, t\right), M^{-1}\left(x, x_{i}, t\right)\right\} \geqslant 1-\varepsilon,
$$

for all $i \geqslant k$, i.e., $\left\{x_{i}\right\}_{i \in D}$ converges strongly to $x \in X$.

The next theorem is the direct conclusion of Proposition 2.6, Lemma 3.5 and Lemma 3.8.

Theorem 3.9. Let $\left\{x_{i}\right\}_{i \in D}$ be a net in a fuzzy metric space $(X, M, *)$. The following conditions are equivalent:

(1) $\left\{x_{i}\right\}_{i \in D}$ converges strongly to $x \in X$.

(2) $\left\{x_{i}\right\}_{i \in D}$ is left K-Cauchy and $x$ is the Yoneda limit of $\left\{x_{i}\right\}_{i \in D}$.

(3) $\left\{x_{i}\right\}_{i \in D}$ is left K-Cauchy and $x$ is the $M^{-1}$-Yoneda limit of $\left\{x_{i}\right\}_{i \in D}$.

The purpose of subsequent part is to introduce and investigate two types of completeness in terms of nets on fuzzy quasi-metric spaces. Many elegant properties in [1,23] for quasi-metric spaces are preserved in our context. Different from the techniques therein, it is worth noting that many proofs depends on the continuity of t-norms and the condition (Fqm-4) in a fuzzy quasi-metric space.

Definition 3.10. A fuzzy quasi-metric space $(X, M, *)$ is said to be:

(1) Yoneda complete, if every left K-Cauchy net $\left\{x_{i}\right\}_{i \in D}$ in $(X, M, *)$ has a Yoneda limit $x \in X$.

(2) Smyth complete, if every left K-Cauchy net $\left\{x_{i}\right\}_{i \in D}$ in $(X, M, *)$ converges strongly to $x \in X$.

The concept of sequentially Yoneda complete in terms of sequences is defined in a natural manner. It is clear that every Yoneda complete fuzzy quasi-metric space is sequentially Yoneda complete.

Example 3.11. Let $\mathfrak{T}(X)$ be a topology on a non-empty set $X$. For each open set $A, B \in \mathfrak{T}(X)$, the fuzzy set $M$ on $\mathfrak{T}(X) \times \mathfrak{T}(X) \times(0,+\infty)$ is given by

$$
M(A, B, t)= \begin{cases}1 & \text { if } A \subseteq B \\ \frac{t}{t+1} & \text { otherwise }\end{cases}
$$


Then, for each continuous t-norm $*,(\mathfrak{T}(X), M, *)$ is a fuzzy quasi-metric space. Each left K-Cauchy net $\left\{A_{i}\right\}_{i \in D}$ in $\mathfrak{T}(X)$ is eventually increasing, which means that there exists $k \in D$ such that $A_{k} \subseteq A_{i} \subseteq A_{j}$ for all $k \leqslant i \leqslant j$. Then we claim that $\bigcup_{i \geqslant k} A_{i}$ is a Yoneda limit of $\left\{A_{i}\right\}_{i \in D}$. Therefore, the fuzzy quasi-metric space $(\mathfrak{T}(\mathrm{X}), \mathrm{M}, *)$ is Yoneda complete.

A direct consequence of Lemma 3.6 will lead to the following statements.

Corollary 3.12. Let $(X, M, *)$ be a fuzzy quasi-metric space. If every left $K$-Cauchy net $\left\{x_{i}\right\}_{i \in D}$ in $(X, M, *)$ has a $\mathrm{M}^{-1}$-Yoneda limit, then $(\mathrm{X}, \mathrm{M}, *)$ is left K-complete.

In particular, every Smyth complete fuzzy quasi-metric space is left K-complete.

The following instance (compare [19, Example 6.4]) provides a sequentially Yoneda complete fuzzy quasi-metric space which is not Yoneda complete.

Example 3.13. Let $\mathcal{A}$ be the family of all non-empty countable subsets of $R$ and $\delta_{0} \in(0,1)$ be a constant. Define a fuzzy set $M$ on $\mathcal{A} \times \mathcal{A} \times(0,+\infty)$ by

$$
M(A, B, t)= \begin{cases}1 & \text { if } A \subseteq B, \\ \delta_{0} & \text { otherwise. }\end{cases}
$$

For each continuous t-norm $*$, the triple $(\mathcal{A}, \mathrm{M}, *)$ is a fuzzy quasi-metric space. It can be easily checked that $(\mathcal{A}, M, *)$ is sequentially Yoneda complete. The subset

$$
\Gamma=\{A: A \text { is a non-empty finite subset of } R \text { consisting of irrational numbers }\},
$$

of $\mathcal{A}$ is directed with the inclusion order and can be regarded itself as a left K-Cauchy net in $(\mathcal{A}, M, *)$. However, the left K-Cauchy net $\Gamma$ does not converge with respect to the topology $\tau_{M^{-1}}$, which implies that $(\mathcal{A}, \mathrm{M}, *)$ is not Yoneda complete by Lemma 3.6.

Lemma 3.14. If a fuzzy quasi-metric space $(X, M, *)$ is Smyth complete, then it is Yoneda complete.

Proof. Suppose that $\left\{x_{i}\right\}_{i \in D}$ is a left $\mathrm{K}-$ Cauchy net in $(X, M, *)$ that converges strongly to $x \in X$. Considering Lemma 3.5, the element $x$ is also a Yoneda limit of $\left\{x_{i}\right\}_{i \in D}$, which implies that $(X, M, *)$ is Yoneda complete.

The following are instances of Smyth complete, in particular, Yoneda complete fuzzy quasi-metric spaces.

Example 3.15. Let $X$ be a finite subset of positive real numbers. Define $a * b=a \cdot b$, the usual product $\mathrm{t}$-norm for every $\mathrm{a}, \mathrm{b} \in[0,1]$ and let $M$ be a fuzzy set on $X \times X \times(0,+\infty)$ defined by

$$
M(x, y, t)= \begin{cases}\frac{x+t}{y+t} & \text { if } x<y \\ 1 & \text { if } x \geqslant y\end{cases}
$$

Then $(X, M, \cdot)$ is a fuzzy quasi-metric space. Since every left K-Cauchy net $\left\{x_{i}\right\}_{i \in D}$ in $X$ is eventually constant, $(X, M, \cdot)$ is Smyth complete.

Example 3.16. Let $X=N$, the set of all positive integers. Define $a * b=a \wedge b$, the minimum t-norm for every $a, b \in[0,1]$. The fuzzy set $M$ on $N \times N \times(0,+\infty)$ is given by

$$
M(m, n, t)= \begin{cases}1, & \text { if } n \leqslant m, \\ \max \left\{\frac{t}{t+1}-\frac{1}{2 m}, \frac{1}{2}\right\} & \text { if } n>m .\end{cases}
$$

It is easy to check that $(X, M, \wedge)$ is a fuzzy quasi-metric space and Smyth complete. 
Lemma 3.17. If a fuzzy quasi-metric space $(X, M, *)$ is sequentially Yoneda complete, then it is bicomplete.

Proof. Let $\left\{x_{i}\right\}_{i \in N}$ be a bi-Cauchy sequence, in particular, a left K-Cauchy sequence in $(X, M, *)$. Since $(X, M, *)$ is sequentially Yoneda complete, $\left\{x_{i}\right\}_{i \in N}$ has a Yoneda limit $x \in X$, which implies $\lim _{i \in N} M\left(x_{i}, x, t\right)=1$ for all $t>0$ by Lemma 3.6. We next show that $\lim _{i \in N} M\left(x, x_{i}, t\right)=1$, for all $t>0$.

Fix $t>0$. For each $\varepsilon \in(0,1)$, since $\left\{x_{i}\right\}_{i \in N}$ is bi-Cauchy in $(X, M, *)$, there exists $n_{0} \in N$ such that $M\left(x_{i}, x_{j}, t\right)>1-\varepsilon$ whenever $i, j \geqslant n_{0}$. Thus we have

$$
\inf _{i \geqslant n_{0}} M\left(x_{i}, x_{j}, t\right) \geqslant 1-\varepsilon,
$$

for any fixed $j \geqslant n_{0}$. It follows from $x$ is the Yoneda limit of $\left\{x_{i}\right\}_{i \in N}$ that

$$
M\left(x, x_{j}, t\right)=\sup _{l \in N} \inf _{i \geqslant l} M\left(x_{i}, x_{j}, t\right) \geqslant \inf _{i \geqslant n_{0}} M\left(x_{i}, x_{j}, t\right) \geqslant 1-\varepsilon,
$$

for any $j \geqslant n_{0}$. Hence, $\lim _{i \in N} M\left(x, x_{i}, t\right)=1$, for all $t>0$.

Therefore, we conclude that $\left\{x_{i}\right\}_{i \in N}$ converges strongly to $x$, i.e., $(X, M, *)$ is bicomplete.

Definition 3.18. Let $c \in X$ be an element in a fuzzy quasi-metric space $(X, M, *)$. For any left $K$-Cauchy net $\left\{x_{i}\right\}_{i \in D}$ with a Yoneda limit $x$ in $(X, M, *)$, if we have

$$
M(c, x, t)=\inf _{j \in D} \sup _{i \geqslant j} M\left(c, x_{i}, t\right),
$$

for all $t>0$, then $c \in X$ is called a finite element.

We have shown that every Smyth complete fuzzy quasi-metric space $(X, M, *)$ is Yoneda complete in Lemma 3.14. Moreover, the notion of finite elements plays an important role in determining the relationship between Smyth completeness and Yoneda completeness.

Theorem 3.19. Let $(X, M, *)$ be a fuzzy quasi-metric space. Then the following conditions are equivalent:

(1) $(\mathrm{X}, \mathrm{M}, *)$ is Smyth complete.

(1) $(X, M, *)$ is Yoneda complete and all elements in $X$ are finite.

Proof. (1) $\Rightarrow(2)$ : We only need to prove that each $c \in X$ is a finite element. Let $\left\{x_{i}\right\}_{i \in D}$ be a left K-Cauchy net in $(X, M, *)$, which has a Yoneda limit $x \in X$. Since $(X, M, *)$ is Smyth complete, $\left\{x_{i}\right\}_{i \in D}$ converges strongly to some $y \in X$. It follows from Proposition 3.4 and Lemma 3.5 that $y=x$. By Proposition 2.6, for each $\varepsilon \in(0,1)$ and $t>0$, there exists $j_{0}$ such that $M^{s}\left(x, x_{i}, \varepsilon t\right)>1-\varepsilon$, in particular, $M\left(x, x_{i}, \varepsilon t\right)>1-\varepsilon$ and $M\left(x_{i}, x, \varepsilon t\right)>1-\varepsilon$, for all $i \geqslant j_{0}$.

(i) For each $j \in D$, we choose a $k \geqslant j$, $j_{0}$, then

$$
M\left(c, x_{k}, t\right) \geqslant M(c, x,(1-\varepsilon) t) * M\left(x, x_{k}, \varepsilon t\right) \geqslant M(c, x,(1-\varepsilon) t) *(1-\varepsilon) .
$$

It follows that

$$
\sup _{i \geqslant j} M\left(c, x_{i}, t\right) \geqslant \sup _{i \geqslant k} M\left(c, x_{i}, t\right) \geqslant M\left(c, x_{k}, t\right) \geqslant M(c, x,(1-\varepsilon) t) *(1-\varepsilon) .
$$

Let $\varepsilon \rightarrow 0$, we have

$$
\sup _{i \geqslant j} M\left(c, x_{i}, t\right) \geqslant M(c, x, t) * 1=M(c, x, t) .
$$

Referring to the free choice of $j \in D$, we obtain that

$$
\inf _{j \in D} \sup _{i \geqslant j} M\left(c, x_{i}, t\right) \geqslant M(c, x, t),
$$

for all $t>0$. 
(ii) Using (Fqm-3), we have

$$
M(c, x,(1+\varepsilon) t) \geqslant M\left(c, x_{i}, t\right) * M\left(x_{i}, x, \varepsilon t\right) \geqslant M\left(c, x_{i}, t\right) *(1-\varepsilon),
$$

for all $i \geqslant j_{0}$. Thus,

$$
M(c, x,(1+\varepsilon) t) \geqslant \sup _{i \geqslant j_{0}} M\left(c, x_{i}, t\right) *(1-\varepsilon) \geqslant\left(\inf _{j \in D} \sup _{i \geqslant j} M\left(c, x_{i}, t\right)\right) *(1-\varepsilon) .
$$

Let $\varepsilon \rightarrow 0$, we conclude that

$$
M(c, x, t) \geqslant\left(\inf _{j \in D} \sup _{i \geqslant j} M\left(c, x_{i}, t\right)\right) * 1=\inf _{j \in D} \sup _{i \geqslant j} M\left(c, x_{i}, t\right),
$$

for all $t>0$.

Therefore, $M(c, x, t)=\inf _{j \in D} \sup _{i \geqslant j} M\left(c, x_{i}, t\right)$ for all $t>0$, i.e., $c$ is a finite element.

$(2) \Rightarrow(1)$ : Let $\left\{x_{i}\right\}_{i \in D}$ be a left K-Cauchy net which has a Yoneda-limit $x$ in $(X, M, *)$. By Lemma 3.6, we have $\lim _{i \in D} M\left(x_{i}, x, t\right)=1$, for all $t>0$.

For each $\varepsilon \in(0,1)$, there exists $\delta \in(0,1)$ satisfying that $(1-\delta) *(1-\delta)>1-\varepsilon$. Since $\left\{x_{i}\right\}_{i \in D}$ is left K-Cauchy, for each $t>0$, there exists $l \in D$ such that $M\left(x_{j}, x_{i}, t / 2\right)>1-\delta$, for all $i \geqslant j \geqslant l$.

Moreover, since all elements in $\mathrm{X}$ are finite,

$$
\inf _{j \in D} \sup _{i \geqslant j} M\left(x, x_{i}, t / 2\right)=M(x, x, t / 2)=1 .
$$

In particular, $\sup _{i \geqslant l} M\left(x, x_{i}, t / 2\right)=1$, for all $t>0$. Then, there exists $k \geqslant l$ such that $M\left(x, x_{k}, t\right)>1-\delta$. Thus, we obtain

$$
M\left(x, x_{i}, t\right) \geqslant M\left(x, x_{k}, t / 2\right) * M\left(x_{k}, x_{i}, t / 2\right) \geqslant(1-\delta) *(1-\delta)>1-\varepsilon,
$$

for all $i \geqslant k$. Therefore, $\left\{x_{i}\right\}_{i \in D}$ converges strongly to $x \in X$.

Remark 3.20. For a fuzzy quasi-metric space $(X, M, *)$, the following diagram in which the arrows mean implication indicates the relations among various completeness on fuzzy quasi-metric spaces.

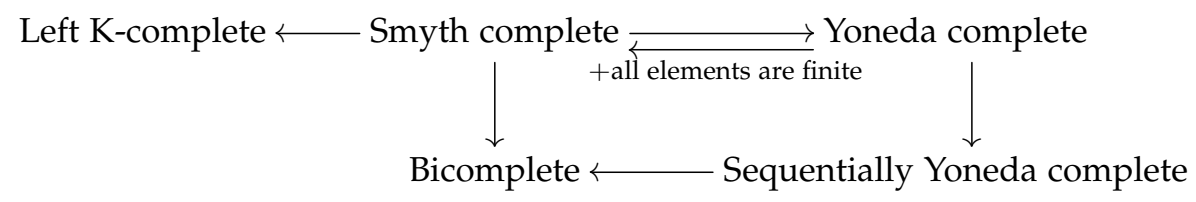

\section{Standard Yoneda completeness and formal balls}

In this section, we turn to consider the relationship between completeness of fuzzy quasi-metric spaces and directed completeness of the associated posets of formal balls.

Let $(P, \leqslant)$ be a poset and $A \subseteq P$ be a non-empty subset. The notation $\bigvee A$ denotes the supremum of $A$, if it exists. A non-empty subset $D \subseteq L$ is called directed, if for each $x, y \in D$, there exists $z \in D$ such that $x \leqslant z$ and $y \leqslant z$. The poset $(\mathrm{P}, \leqslant)$ is a dcpo (directed complete poset), if every directed subset $\mathrm{D} \subseteq \mathrm{P}$ has a supremum $\bigvee D$ in $P$. For more details, the reader can refer to [4].

A formal ball in a fuzzy quasi-metric space $(X, M, *)$ is a pair $(x, r)$ with $x \in X, r \in[0,+\infty)$, the set of all formal balls is denoted by $\mathrm{BX}=\mathrm{X} \times[0,+\infty)$. In [20], Ricarte and Romaguera defined a binary relation on $\mathrm{BX} \times \mathrm{BX}$ by

$$
(x, r) \sqsubseteq_{M}(y, s) \text { if and only if } M(x, y, t) \geqslant \frac{t}{t+r-s} \text { for all } t>0 \text {. }
$$


For a fuzzy quasi-metric space $(X, M, *)$, if the continuous t-norm $*=\wedge$, then the binary relation $\Xi_{M}$ is a partial order on BX. Moreover, a directed subset of $B X$ in $(X, M, \wedge)$ is denoted by $\Gamma=\left\{\left(x_{i}, r_{i}\right)\right\}_{i \in D}$, where $D$ is a directed set and $\left(x_{i}, r_{i}\right) \sqsubseteq_{M}\left(x_{j}, r_{j}\right)$, if and only if $i \leqslant j$ for each $i, j \in D$.

Proposition 4.1. Let $(X, M, \wedge)$ be a fuzzy quasi-metric space. Then $(B X, \sqsubseteq M)$ is a poset.

Remark 4.2. If $(x, r) \sqsubseteq_{M}(y, s)$, then we have $r \geqslant s$.

In [19], Ricarte gave the notion of standard completeness on KM-fuzzy quasi-metric spaces in terms of left K-Cauchy sequences and constructed a counterexample of standard complete KM-fuzzy quasi-metric spaces $(X, M, \wedge)$ to show that the poset $\left(B X, \sqsubseteq_{M}\right)$ is not a dcpo. The reader can refer to $[2,8,19,21]$ for more details about the KM-fuzzy quasi-metric spaces. Now we review and adapt the concept of standard completeness to fuzzy quasi-metric spaces considered in this paper. The slight differences are that we use left K-Cauchy nets instead of left K-Cauchy sequences and focus on fuzzy quasi-metric spaces instead of KM-fuzzy quasi-metric spaces.

Definition 4.3. Let $(X, M, \wedge)$ be a fuzzy quasi-metric space. A net $\left\{x_{i}\right\}_{i \in D}$ is said to be standard left K-Cauchy, if for each $\varepsilon \in(0,1)$, there exists $k_{\varepsilon} \in D$ such that $M\left(x_{i}, x_{j}, t\right) \geqslant \frac{t}{t+\varepsilon}$, for all $j \geqslant i \geqslant k_{\varepsilon}$ and $t>0$. A fuzzy quasi-metric space $(X, M, \wedge)$ is called standard complete, if every standard left K-Cauchy net converges with respect to the topology $\tau_{M^{-1}}$.

Then a related problem arises from [19, Chapter 6] as the following statement.

Problem 4.4. When does the poset of formal balls $\left(B X, \sqsubseteq_{M}\right)$ in a fuzzy quasi-metric space $(X, M, \wedge)$ constitutes a dcpo?

In the subsequent part, we will focus on this problem. Since the concept of a standard left K-Cauchy net is uniformly depended on $\mathrm{t}>0$, every standard left $\mathrm{K}$-Cauchy net is a left $\mathrm{K}$-Cauchy net. Furthermore, in order to deal with Problem 4.4, we only need to require that every standard left K-Cauchy net in a fuzzy quasi-metric space has a Yoneda limit.

Definition 4.5. A fuzzy quasi-metric space $(X, M, \wedge)$ is called standard Yoneda complete, if every standard left K-Cauchy net $\left\{x_{i}\right\}_{i \in D}$ has a Yoneda limit $x \in X$.

The fuzzy quasi-metric spaces of Examples 3.11, 3.15 and 3.16 are all standard Yoneda complete. The notion of standard sequentially Yoneda complete is defined in an obvious manner. By Lemma 3.6, every standard Yoneda complete fuzzy quasi-metric space is standard complete.

We denote the Sorgenfrey topology on $R$ (the set of all real numbers) by $\tau_{S}$. It is well-known that the quasi-metric $\mathrm{d}$ defined on $\mathrm{R}$ by

$$
d(x, y)= \begin{cases}y-x & \text { if } x \leqslant y \\ 1 & \text { if } x>y\end{cases}
$$

generates the Sorgenfrey topology $\tau_{S}$. In [6], the authors proved that the Sorgenfrey line does not admit any fuzzy quasi-metric $M$ such that $(R, M, *)$ is left $\mathrm{K}$-sequentially complete and $\tau_{M}=\tau_{S}$.

Example 4.6 (Sorgenfrey line). Let $R$ be the set of real numbers. The fuzzy quasi-metric $M_{d}$ on $R$ is defined for all $x, y \in R$ and $t>0$ by

$$
M_{d}(x, y, t)= \begin{cases}\frac{t}{t+y-x} & \text { if } x \leqslant y, \\ \frac{t}{t+1} & \text { if } x>y .\end{cases}
$$

By Example 2.4, we get $\tau_{M_{d}}=\tau_{S}$. It can be easily checked that the notions of standard left K-Cauchy nets and standard Yoneda complete on the induced standard fuzzy quasi-metric space $\left(X, M_{d}, *\right)$ coincide 
with that of Cauchy nets and Yoneda complete defined in [1] on a quasi-metric space $(X, d)$, respectively. As it has been shown in [1, Example 2.4] that the Sorgenfrey line $(R, d)$ is Yoneda complete, we obtain that Sorgenfrey line admit the fuzzy quasi-metric $M_{d}$ such that $\left(R, M_{d}, *\right)$ is standard Yoneda complete and $\tau_{M_{\mathrm{d}}}=\tau_{\mathrm{S}}$.

Lemma 4.7. Let $(\mathrm{X}, \mathrm{M}, \wedge)$ be a fuzzy quasi-metric space. Suppose that the poset $\left(\mathrm{BX}, \sqsubseteq_{M}\right)$ is a dcpo and $\Gamma=$ $\left\{\left(x_{i}, r_{i}\right)\right\}_{i \in D}$ is a directed subset of $B X$ such that $\bigvee \Gamma=(x, r)$. Then we have $\bigvee_{i \in D}\left(x_{i}, r_{i}+\theta\right)=(x, r+\theta)$, for any $\theta \geqslant 0$.

Proof. Let $\Gamma_{\theta}=\left\{\left(x_{i}, r_{i}+\theta\right)\right\}_{i \in D}$. Since $\bigvee \Gamma=(x, r)$, for each $i \in D$, we obtain

$$
M\left(x_{i}, x, t\right) \geqslant \frac{t}{t+r_{i}-r}=\frac{t}{t+\left(r_{i}+\theta\right)-(r+\theta)},
$$

for all $t>0$. Then, $\left(x_{i}, r_{i}+\theta\right) \sqsubseteq M(x, r+\theta)$ for each $i \in D$. Thus, $(x, r+\theta)$ is an upper bound of $\Gamma_{\theta}$.

Since $\Gamma_{\theta}$ is directed and $B X$ is a dcpo, $\bigvee \Gamma_{\theta}=(y, s)$ exists. Next, we will prove that $(x, r+\theta)=(y, s)$. Obviously, we have $(y, s) \sqsubseteq_{M}(x, r+\theta)$.

Conversely, since $(y, s)$ is an upper bound of $\Gamma_{\theta}$, we have

$$
M\left(x_{i}, y, t\right) \geqslant \frac{t}{t+r_{i}+\theta-s}=\frac{t}{t+r_{i}-(s-\theta)},
$$

for all $t>0$. Thus, $\left(x_{i}, r_{i}\right) \sqsubseteq_{M}(y, s-\theta)$ for each $i \in D$, which follows that $(x, r)=\bigvee \Gamma \sqsubseteq_{M}(y, s-\theta)$ Then, we get

$$
M(x, y, t) \geqslant \frac{t}{t+r-(s-\theta)}=\frac{t}{t+(r+\theta)-s}
$$

for all $t>0$. Therefore, $(x, r+\theta) \sqsubseteq_{M}(y, s)$ which implies $(y, s)=(x, r+\theta)$.

Theorem 4.8. Let $(\mathrm{X}, \mathrm{M}, \wedge)$ be a fuzzy quasi-metric space. If $(\mathrm{BX}, \sqsubseteq \mathrm{M})$ is a dcpo and $\Gamma=\left\{\left(\mathrm{x}_{\mathrm{i}}, \mathrm{r}_{\mathrm{i}}\right)\right\}_{i \in \mathrm{D}}$ is a directed subset of $B X$ such that $\bigvee \Gamma=(x, r)$, then $r=\inf \left\{r_{i}\right\}_{i \in D}$ and $\left\{x_{i}\right\}_{i \in D}$ converges to $x$ with respect to the topology $\tau_{M^{-1}}$.

Proof. Suppose $s=\inf \left\{r_{i}\right\}_{i \in D}$. We first prove that $r=s$. Since BX is a dcpo and $\Gamma_{s}=\left\{\left(x_{i}, r_{i}-s\right)\right\}_{i \in D}$ is clearly directed, there exists $(y, l) \in B X$ such that $\bigvee \Gamma_{s}=(y, l)$. Then

$$
l \leqslant \inf \left\{r_{i}-s\right\}_{i \in D}=\inf \left\{r_{i}\right\}_{i \in D}-s=s-s=0,
$$

by Remark 4.2. Thus $l=0$. It follows from Lemma 4.7 that $(x, r)=\bigvee \Gamma=(y, l+s)=(y, s)$. So $r=s=\inf \left\{r_{i}\right\}_{i \in D}$.

For each $\varepsilon \in(0,1)$ and $t>0$, let $0<\varepsilon_{1}<\frac{t \varepsilon}{1-\varepsilon}$. Since $r=\inf \left\{r_{i}\right\}_{i \in D}$, there exists an $i_{0} \in D$ such that $r_{i_{0}}<r+\varepsilon_{1}$. Because $(x, r)$ is an upper bound of $\Gamma$, we obtain that $M\left(x_{i}, x, u\right) \geqslant \frac{u}{u+r_{i}-r}$, for all $u>0$. In particular for all $i \geqslant i_{0}$, we have

$$
M\left(x_{i}, x, t\right) \geqslant \frac{t}{t+r_{i}-r}>\frac{t}{t+r_{i_{0}}-r}>\frac{t}{t+\varepsilon_{1}}>1-\varepsilon .
$$

Therefore, the net $\left\{x_{i}\right\}_{i \in D}$ converges to $x$ with respect to the topology $\tau_{M^{-1}}$.

Then we immediately obtain the following result.

Corollary 4.9. Let $(\mathrm{X}, \mathrm{M}, \wedge)$ be a fuzzy quasi-metric space. If $\left(\mathrm{BX}, \sqsubseteq_{\mathrm{M}}\right)$ is a dcpo, then $\mathrm{X}$ is standard complete. 
A natural question is whether the converse of Corollary 4.9 is correct. First of all, we give the following two examples.

Example 4.10. Let $\mathcal{F}$ be the family of all non-empty finite subsets of $R$ and $\delta_{0} \in(0,1)$ be a constant. Define a fuzzy set $M$ on $\mathcal{F} \times \mathcal{F} \times(0,+\infty)$ by

$$
M(A, B, t)= \begin{cases}1 & \text { if } A=B, \\ \delta_{0} & \text { otherwise. }\end{cases}
$$

Then the triple $(\mathcal{F}, M, \wedge)$ is a fuzzy (quasi-)metric space. It is almost obvious that $(\mathcal{F}, M, \wedge)$ is standard complete. For each directed subset $\Gamma=\left\{\left(F_{i}, r_{i}\right)\right\}_{i \in D}$ of $\left(B X, \sqsubseteq_{M}\right)$, we obtain that $F_{i}=F_{j}$, for all $i, j \in D$ and $\bigvee \Gamma=\left(F_{i_{0}}, \inf \left\{r_{i}\right\}_{i \in D}\right)$ for an $i_{0} \in D$. Thus the poset $\left(B \mathcal{F}, \sqsubseteq_{M}\right)$ is a dcpo.

Let us recall that a topological space $(X, \mathfrak{T})$ is compact, if and only if any collection of closed subsets of $X$ with the finite intersection property has non-empty intersection.

Example 4.11. Given a compact topological space $(X, \mathfrak{T})$ and a family of non-empty closed subsets $\left\{C_{i}\right\}_{i \in I}$ with the finite intersection property. Let $\mathcal{C}$ be a family consisting of all $C_{i}$ and their arbitrary intersections. That is, $\mathcal{C}=\left\{\mathrm{C}_{i}\right\}_{i \in \mathrm{I}} \cup\left\{\bigcap_{\mathrm{K} \in \mathrm{K}} \mathrm{C}_{\mathrm{k}} \mid \mathrm{K} \subseteq \mathrm{I}\right\}$. Define a fuzzy set $M$ on $\mathcal{C} \times \mathcal{C} \times(0,+\infty)$ by

$$
M(A, B, t)= \begin{cases}1 & \text { if } B \subseteq A, \\ \min \left\{t, \frac{1}{2}\right\} & \text { otherwise }\end{cases}
$$

Then the triple $(\mathcal{C}, M, \wedge)$ is a fuzzy quasi-metric space. If $\left\{B_{i}\right\}_{i \in D}$ is a standard left $\mathrm{K}$-Cauchy net in $(\mathcal{C}, M, \wedge)$, then $\left\{B_{i}\right\}_{i \in D}$ is eventually decreasing and converges to $\bigcap_{i \in D} B_{i} \neq \emptyset$ with respect to the topology $\tau_{M^{-1}}$. Therefore, the fuzzy quasi-metric space $(\mathcal{C}, M, \wedge)$ is standard complete.

For each directed subset $\Gamma=\left\{\left(B_{i}, r_{i}\right)\right\}_{i \in D}$ of $(B X, \sqsubseteq M)$, it has a supremum $\bigvee \Gamma=\left(\bigcap_{i \in D} B_{i}, \inf \left\{r_{i}\right\}_{i \in D}\right)$. Thus, the poset $\left(\mathrm{BC}, \sqsubseteq_{M}\right)$ is a dcpo.

However, a further examination shows that the above two fuzzy quasi-metric spaces are not only standard complete, but also standard Yoneda complete. Next, we want to show that the answer of the converse of Corollary 4.9 is "yes" when $(X, M, \wedge)$ is a standard Yoneda complete fuzzy quasi-metric space.

Proposition 4.12. Let $(X, M, \wedge)$ be a fuzzy quasi-metric space and $\Gamma=\left(x_{i}, r_{i}\right)_{i \in D}$ be a directed subset of $B X$. Then $\left\{x_{i}\right\}_{i \in D}$ is a standard left K-Cauchy net.

Proof. Suppose $r=\inf \left\{r_{i}\right\}_{i \in D}$. For each $\varepsilon>0$, there exists $i_{\varepsilon} \in D$ such that $r_{i_{\varepsilon}}<r+\varepsilon$, i.e., $r_{i_{\varepsilon}}-r<\varepsilon$. For any $i, j \in D$ satisfying $j \geqslant i \geqslant i_{\varepsilon}$, we have $\left(x_{i_{\varepsilon}}, r_{i_{\varepsilon}}\right) \sqsubseteq_{M}\left(x_{i}, r_{i}\right) \sqsubseteq_{M}\left(x_{j}, r_{j}\right)$, which implies that $r_{i_{\varepsilon}} \geqslant r_{i} \geqslant r_{j}$. Hence,

$$
M\left(x_{i}, x_{j}, t\right) \geqslant \frac{t}{t+r_{i}-r_{j}} \geqslant \frac{t}{t+r_{i_{\varepsilon}}-r_{j}} \geqslant \frac{t}{t+r_{i_{\varepsilon}}-r}>\frac{t}{t+\varepsilon},
$$

for all $j \geqslant i \geqslant i_{\varepsilon}$ and $t>0$. Therefore, $\left\{x_{i}\right\}_{i \in D}$ is a standard left K-Cauchy net.

Lemma 4.13. Let $(X, M, \wedge)$ be a fuzzy quasi-metric space and $\Gamma=\left\{\left(x_{i}, r_{i}\right)\right\}_{i \in D}$ be a directed subset of $B X$. If $x$ is a Yoneda limit of the net $\left\{x_{i}\right\}_{i \in D}$ and $r=\inf \left\{r_{i}\right\}_{i \in D}$, then $\bigvee \Gamma=(x, r)$.

Proof. We first show that $(x, r)$ is an upper bound of $\left\{\left(x_{i}, r_{i}\right)\right\}_{i \in D}$. For each $\varepsilon \in(0,1)$ and $t>0$, since $x$ is a Yoneda limit of $\left\{x_{i}\right\}_{i \in D}$, we have $\sup _{j \in D} \inf _{i \geqslant j} M\left(x_{i}, x, \varepsilon t\right)=M(x, x, \varepsilon t)=1$. Hence, there exits $j_{0} \in D$ such that $M\left(x_{k}, x, \varepsilon t\right)>1-\varepsilon$, for all $k \geqslant j_{0}$. For each $i \in D$, we choose a $k_{0} \geqslant i, j_{0} \in D$, then

$$
M\left(x_{i}, x_{k_{0}}, u\right) \geqslant \frac{u}{u+r_{i}-r_{k_{0}}},
$$


for all $u>0$, in particular, $M\left(x_{i}, x_{k_{0}}, t\right) \geqslant \frac{t}{t+r_{i}-r_{k_{0}}}$. Thus, we conclude that

$$
\begin{aligned}
M\left(x_{i}, x_{,}(1+\varepsilon) t\right) & \geqslant M\left(x_{i}, x_{k_{0}}, t\right) \wedge M\left(x_{k_{0}}, x, \varepsilon t\right) \geqslant\left(\frac{t}{t+r_{i}-r_{k_{0}}}\right) \wedge(1-\varepsilon) \\
& \geqslant\left(\frac{t}{t+r_{i}-r}\right) \wedge(1-\varepsilon) .
\end{aligned}
$$

Let $\varepsilon \rightarrow 0$, we obtain

$$
M\left(x_{i}, x, t\right)=\lim _{\varepsilon \rightarrow 0} M\left(x_{i}, x,(1+\varepsilon) t\right) \geqslant \lim _{\varepsilon \rightarrow 0}\left(\frac{t}{t+r_{i}-r}\right) \wedge(1-\varepsilon)=\frac{t}{t+r_{i}-r^{\prime}},
$$

for all $t>0$, which follows that $\left(x_{i}, r_{i}\right) \sqsubseteq_{M}(x, r)$ for each $i \in D$.

Assume that $(y, l)$ is another upper bound of $\Gamma$. For each $\left(x_{i}, r_{i}\right) \in \Gamma$, we have $M\left(x_{i}, y, t\right) \geqslant \frac{t}{t+r_{i}-l}$ for all $t>0$. Since $x$ is a Yoneda limit of $\left\{x_{i}\right\}_{i \in D}$ and $r=\inf \left\{r_{i}\right\}_{i \in D}$, we get

$$
\begin{aligned}
M(x, y, t) & =\sup _{j \in D} \inf _{i \geqslant j} M\left(x_{i}, y, t\right) \geqslant \sup _{j \in D} \inf _{i \geqslant j}\left(\frac{t}{t+r_{i}-l}\right) \\
& =\sup _{j \in D}\left(\frac{t}{t+r_{j}-l}\right)=\frac{t}{t+r-l^{\prime}}
\end{aligned}
$$

for all $t>0$, i.e., $(x, r) \sqsubseteq_{M}(y, l)$. Therefore, $\bigvee \Gamma=(x, r)$.

Theorem 4.14. If the fuzzy quasi-metric space $(X, M, \wedge)$ is standard Yoneda complete, then the poset $(B X, \sqsubseteq M)$ is a dcpo.

Proof. Let $\Gamma=\left\{\left(x_{i}, r_{i}\right)\right\}_{i \in D}$ be any directed subset of BX and we denote $r=\inf \left\{r_{i}\right\}_{i \in D}$. Then $\left\{x_{i}\right\}_{i \in D}$ is standard left K-Cauchy by Proposition 4.12. Since $X$ is standard Yoneda complete, the net $\left\{x_{i}\right\}_{i \in D}$ has a Yoneda limit $x$. Applying Lemma 4.13, we have $\bigvee \Gamma=(x, r)$.

Corollary 4.15. For a Yoneda complete fuzzy quasi-metric space $(\mathrm{X}, \mathrm{M}, \wedge)$, the poset $\left(\mathrm{BX}, \sqsubseteq_{\mathrm{M}}\right)$ is a dcpo. In particular, the conclusion holds for any Smyth complete fuzzy quasi-metric space.

In the following, we give a characterization of three types of completeness on a fuzzy metric space $(X, M, \wedge)$ by the associated poset $\left(B X, \sqsubseteq_{M}\right)$. The result provides more insight to the study of the interplay between the fuzzy metric spaces and the order structures of formal balls.

Theorem 4.16. Let $(X, M, \wedge)$ be a fuzzy metric space. Then the following conditions are equivalent:

(1) $(X, M, \wedge)$ is standard Yoneda complete.

(2) $(X, M, \wedge)$ is standard sequentially Yoneda complete.

(3) $(X, M, \wedge)$ is standard complete.

(4) $\left(\mathrm{BX}, \sqsubseteq_{\mathrm{M}}\right)$ is a dcpo.

Proof. (1) $\Rightarrow(4)$ and (4) $\Rightarrow(3)$ : The conclusions of Proposition 4.14 and Corollary 4.9.

(3) $\Rightarrow(1)$ : Suppose that $\left\{x_{i}\right\}_{i \in D}$ is a standard left $K$-Cauchy net in $(X, M, \wedge)$. Since $(X, M, \wedge)$ is standard complete, $\left\{x_{i}\right\}_{i \in D}$ converges to some $x \in X$ with respect to the topology $\tau_{M^{-1}}=\tau_{M}$. Thus, $\left\{x_{i}\right\}_{i \in D}$ converges strongly to some $x \in X$. By Theorem 3.9, we obtain that $x$ is a Yoneda limit of $\left\{x_{i}\right\}_{i \in D}$.

$(1) \Rightarrow(2)$ : Straightforward.

(2) $\Rightarrow(3)$ : Let $\left\{x_{i}\right\}_{i \in D}$ be a standard left $\mathrm{K}$-Cauchy net in $(X, M, \wedge)$. Then we can construct a sequence (not always a subsequence) $\left\{x_{i_{k}}\right\}_{k \in N}$ from the net $\left\{x_{i}\right\}_{i \in D}$ with $i_{k+1} \geqslant i_{k} \in D$ for each $k \in N$, satisfying that

$$
M\left(x_{i}, x_{j}, t\right)>\frac{t}{t+2^{-k}}
$$


for all $i_{k} \leqslant i \leqslant j$ and $t>0$.

Next, we will show that $\left\{x_{i}\right\}_{i \in D}$ converges to $x$ with respect to the topology $\tau_{M^{-1}}=\tau_{M}$.

Fix an arbitrary $s>0$. For each $\varepsilon \in(0,1)$, there exists $k_{s, \varepsilon}^{1} \in N$ such that $0<2^{-k}<\frac{s \varepsilon}{1-\varepsilon}$ whenever $k \geqslant k_{s, \varepsilon}^{1}$. Thus, for each $k \geqslant k_{s, \varepsilon}^{1} \in N$ and $i_{k} \leqslant j$, we have

$$
M\left(x_{i_{k}}, x_{j}, t\right)>\frac{t}{t+2^{-k}}>\frac{t}{t+s \varepsilon /(1-\varepsilon)},
$$

for all $t>0$. In particular,

$$
M\left(x_{i_{k}}, x_{j}, s\right)>\frac{s}{s+s \varepsilon /(1-\varepsilon)}=1-\varepsilon,
$$

holds for each $k \geqslant k_{s, \varepsilon}^{1}$ and $j \geqslant i_{k}$.

Moreover, we observe that $\left\{x_{i_{k}}\right\}_{k \in N}$ is a standard left K-Cauchy sequence. Hence, $\left\{x_{i_{k}}\right\}_{k \in N}$ has a Yoneda limit $x \in X$. By Lemma 3.6, there exists $k_{s, \varepsilon}^{2} \in N$ such that $M\left(x, x_{i_{k}}, s\right)=M\left(x_{i_{k}}, x, s\right)>1-\varepsilon$ whenever $k \geqslant k_{s, \varepsilon}^{2}$.

Hence, there exists a $k_{0} \geqslant k_{s, \varepsilon}^{1}, k_{s, \varepsilon}^{2} \in N$ such that

$$
M\left(x, x_{j}, 2 s\right) \geqslant M\left(x, x_{i_{k_{0}}}, s\right) \wedge M\left(x_{i_{k_{0}}}, x_{j}, s\right)>(1-\varepsilon) \wedge(1-\varepsilon)=1-\varepsilon,
$$

for all $j \geqslant i_{k_{0}}$. Since $s>0$ is arbitrarily selected, this finishes the proof.

Remark 4.17. In Theorem 4.14, we give an answer to Problem 4.4. Moreover, we note that the equivalence of (3) and (4) in Theorem 4.16 can also be proved by using the result of [20, Theorem 1]. In this paper, we obtain this result through a different method and show that the standard Yoneda completeness and standard sequentially Yoneda completeness on a fuzzy metric space $(X, M, \wedge)$ can also be characterized by the poset $\left(B X, \sqsubseteq_{M}\right)$ being a dcpo.

\section{Conclusions and questions}

In this paper, we introduce two types of completeness on fuzzy quasi-metric space and show the capability of standard Yoneda completeness in the study of order-theoretical properties of formal balls. It has been proved that if a fuzzy quasi-metric space $(X, M, \wedge)$ is standard Yoneda complete, then the poset of formal balls $\left(B X, \sqsubseteq_{M}\right)$ is a dcpo. Furthermore, we give an order-theoretical characterization of three types of completeness on a fuzzy metric space.

The following are some questions we want to study in the future.

Question 1 Is a fuzzy quasi-metric space $(X, M, \wedge)$ standard Yoneda complete under the assumption that the associated poset $\left(\mathrm{BX}, \sqsubseteq_{M}\right)$ of formal balls is a dcpo?

Question 2 How to construct a satisfactory Yoneda completion for any fuzzy quasi-metric space in the sense of [22]?

\section{Acknowledgment}

The authors are grateful to the anonymous reviewers and the area editor for their careful reading and comments. This work is supported by the NSFC (No. 11371130, 11401195), the Research Fund for the Doctoral Program of Higher Education of China (No. 20120161110017) and the Hunan Provincial Natural Science Foundation of China (No. 2015JJ3050).

\section{References}

[1] M. Ali-Akbari, B. Honari, M. Pourmahdian, M. M. Rezaii, The space of formal balls and models of quasi-metric spaces, Math. Structures Comput. Sci., 19 (2009), 337-355. 1, 3, 4.6

[2] F. Castro-Company, S. Romaguera, P. Tirado, The bicompletion of fuzzy quasi-metric spaces, Fuzzy Sets and Systems, 166 (2011), 56-64.2.4, 2.5, 2, 4 
[3] A. George, P. Veeramani, On some results in fuzzy metric spaces, Fuzzy Sets and Systems, 64 (1994), 395-399. 1, 2, 2.2

[4] J. Goubault-Larrecq, Non-Hausdorff topology and domain theory, [On the cover: Selected topics in point-set topology], New Mathematical Monographs, Cambridge University Press, Cambridge, (2013). 3, 4

[5] V. Gregori, J. A. Mascarell, A. Sapena, On completion of fuzzy quasi-metric spaces, Topology Appl., 153 (2005), 886-899. 1

[6] V. Gregori, S. Morillas, B. Roig, Fuzzy quasi-metrics for the Sorgenfrey line, Fuzzy Sets and Systems, 222 (2013), 98-107. 1, 2.5, 2, 4

[7] V. Gregori, S. Morillas, A. Sapena, Examples of fuzzy metrics and applications, Fuzzy Sets and Systems, 170 (2011), 95-111. 1

[8] V. Gregori, S. Romaguera, Fuzzy quasi-metric spaces, Appl. Gen. Topol., 5 (2004), 129-136. 1, 2.1, 2.2, 2.4, 4

[9] V. Gregori, S. Romaguera, A. Sapena, A characterization of bicompletable fuzzy quasi-metric spaces, Fuzzy Sets and Systems, 152 (2005), 395-402. 1, 2

[10] J. Gutiérrez García, M. A. de Prada Vicente, Hutton [0,1]-quasi-uniformities induced by fuzzy (quasi)-metric spaces, Fuzzy Sets and Systems, 157 (2006), 755-766. 1

[11] M. Kostanek, P. Waszkiewicz, The formal ball model for Q-categories, Math. Structures Comput. Sci., 21 (2011), 41-64. 1

[12] I. Kramosil, J. Michálek, Fuzzy metrics and statistical metric spaces, Kybernetika (Prague), 11 (1975), 336-344. 1

[13] I. Mardones-Pérez, M. A. de Prada Vicente, Fuzzy pseudometric spaces vs fuzzifying structures, Fuzzy Sets and Systems, 267 (2015), 117-132. 1

[14] I. Mardones-Pérez, M. A. de Prada Vicente, An application of a representation theorem for fuzzy metrics to domain theory, Fuzzy Sets and Systems, 300 (2016), 72-83. 1

[15] D. Mihet, Fuzzy quasi-metric versions of a theorem of Gregori and Sapena, Iran. J. Fuzzy Syst., 7 (2010), 59-64. 1

[16] J. Miñana, A. Šostak, Fuzzifying topology induced by a strong fuzzy metric, Fuzzy Sets and Systems, 300 (2016), 24-39. 1

[17] D. Qiu, W.-Q. Zhang, C. Li, Extension of a class of decomposable measures using fuzzy pseudometrics, Fuzzy Sets and Systems, 222 (2013), 33-44. 1

[18] D. Qiu, W.-Q. Zhang, C. Li, On decomposable measures constructed by using stationary fuzzy pseudo-ultrametrics, Int. J. Gen. Syst., 42 (2013), 395-404. 1

[19] L. A. Ricarte, Topological and computational models for fuzzy metric spaces via domain theory, Ph.D. thesis, University Politècnica de València, (2013). 1, 3, 4, 4

[20] L. A. Ricarte, S. Romaguera, A domain-theoretic approach to fuzzy metric spaces, Topology Appl., 163 (2014), $149-159$. $1,4,4.17$

[21] J. Rodríguez-López, S. Romaguera, J. M. Sánchez-Álvarez, The Hausdorff fuzzy quasi-metric, Fuzzy Sets and Systems, 161 (2010), 1078-1096. 3, 4

[22] S. Romaguera, A. Sapena, O. Valero, Quasi-uniform isomorphisms in fuzzy quasi-metric spaces, bicompletion and Dcompletion, Acta Math. Hungar., 114 (2007), 49-60. 1, 2, 2

[23] S. Romaguera, O. Valero, Domain theoretic characterisations of quasi-metric completeness in terms of formal balls, Math. Structures Comput. Sci., 20 (2010), 453-472. 1, 3

[24] A. Savchenko, M. Zarichnyi, Fuzzy ultrametrics on the set of probability measures, Topology, 48 (2009), 130-136. 1

[25] Y.-H. Shen, D. Qiu, W. Chen, Fixed point theorems in fuzzy metric spaces, Appl. Math. Lett., 25 (2012), 138-141. 1

[26] J.-Y. Wu, Y.-L. Yue, Formal balls in fuzzy partial metric spaces, Iran. J. Fuzzy Syst., (2016), Article in press. 1

[27] J.-L. Xie, Q.-G. Li, S.-L. Chen, Y. Gao, On pseudo-metric spaces induced by $\sigma$ - $\perp$-decomposable measures, Fuzzy Sets and Systems, 289 (2016), 33-42. 1

[28] Y.-L. Yue, F.-G. Shi, On fuzzy pseudo-metric spaces, Fuzzy Sets and Systems, 161 (2010), 1105-1116. 1 